\title{
Osteotomien des Unterschenkels nach fehlverheilten Frakturen
}

\author{
F. Holz, A. Wentzensen, V. Heppert
}

\section{Zusammenfassung}

In Achsfehlstellung verheilte Unterschenkelfrakturen oder fehlverheilte Tibiakopffrakturen führen unweigerlich über Fehlbelastung eines Gelenkkompartiments zur fortschreitenden Kniegelenksarthrose, das gleiche gilt für Fehlstellungen des Sprunggelenkes. Je nach Ort der Fehlstellung und vorliegender Weichteildeckung bieten sich verschiedene Korrekturoperationen mit dem Ziel der Entlastung des teilzerstörten Gelenkabschnittes, Entlastung des Kapsel-Band-Apparates sowie auch einer Besserung des subjektiven Beschwerdebildes an. Die Korrekturmaßnahme soll zu einer Verlangsamung, bei rechtzeitigem Eingreifen zum Stopp des degenerativen Umbaus der Fehlstellung benachbarter Gelenkabschnitte führen. Prinzi- piell unterscheidet man Osteotomien im metaphysären oder diaphysären Bereich. Wenn möglich, sollten Osteotomien im metaphysären Bereich auf Grund der besseren knöchernen Heilung durchgeführt werden. Eine exakte Fehlstellungsanalyse bez. aller räumlicher Ebenen ist unerlässlich. Neben einer klinischen Untersuchung, die auch auf scheinbare Fehlstellungen achten muss, ist eine ausgiebige radiologische Diagnostik mit Ganzbeinaufnahmen, ggf. ergänzt durch computertomographische Untersuchungen erforderlich. Eine breite und differenzierte präoperative Aufklärung muss erfolgen, dem Verletzten muss eine realistische Erwartungshaltung vermittelt werden. Korrekturosteotomien setzen einen erfahren Operateur voraus.

Bei schwerem Weichteilschaden erfordert das Debridement neben der Entfernung avitaler Weichteile auch die Entfernung avaskulärer Knochensegmente. Unter Umständen kann damit ein primärer Defekt gedeckt und eine sichere Knochenbruchheilung erzielt werden, unter Verzicht auf primär korrekten Längenausgleich.

Frakturheilungsstörungen mit Knochenresorption im Frakturbereich können ebenso zur sekundären Fehlstellung führen.

Fehlstellungen können prinzipiell bei allen internen oder externen Osteosyntheseverfahren auftreten, speziell bei der Marknagelung muß hier auch mit sekundären Torsionsfehlern gerechnet werden, wobei intraoperativ die Torsion am Unterschenkel klinisch erheblich einfacher und suffizienter als am Oberschenkel zu kontrollieren ist.

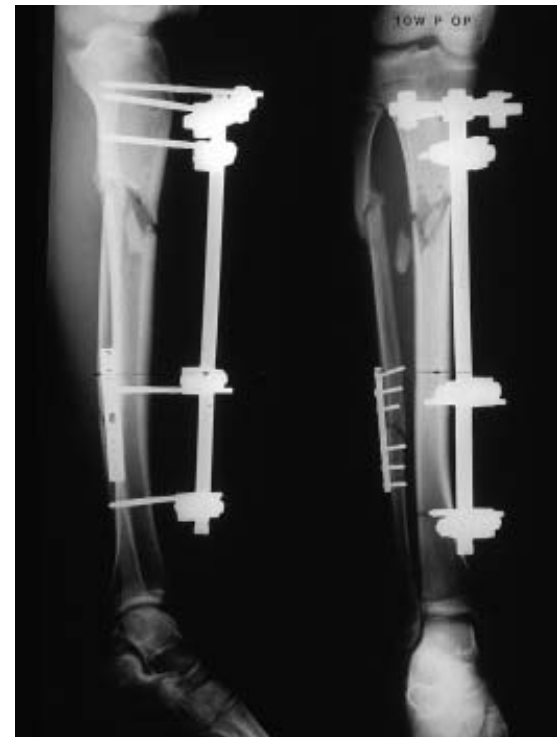

Abb.1a Mit Fixateur externe versorgte offene Unterschenkelfraktur.

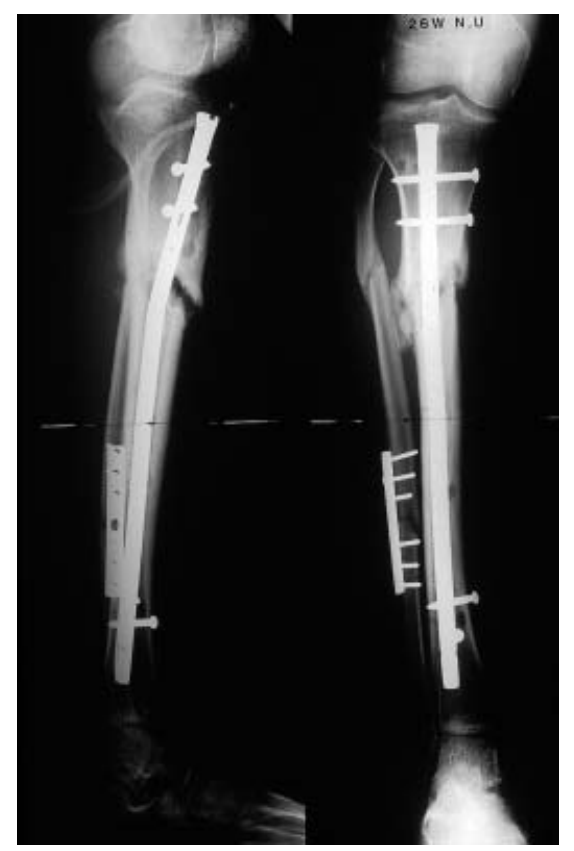

Abb.1b Verfahrenswechsel auf gebohrten Marknagel nach Weichteilkonditionierung mit zunehmender Varusstellung.
OP-JOURNAL 2000; 16: 61-67

(C) Georg Thieme Verlag Stuttgart . New York 


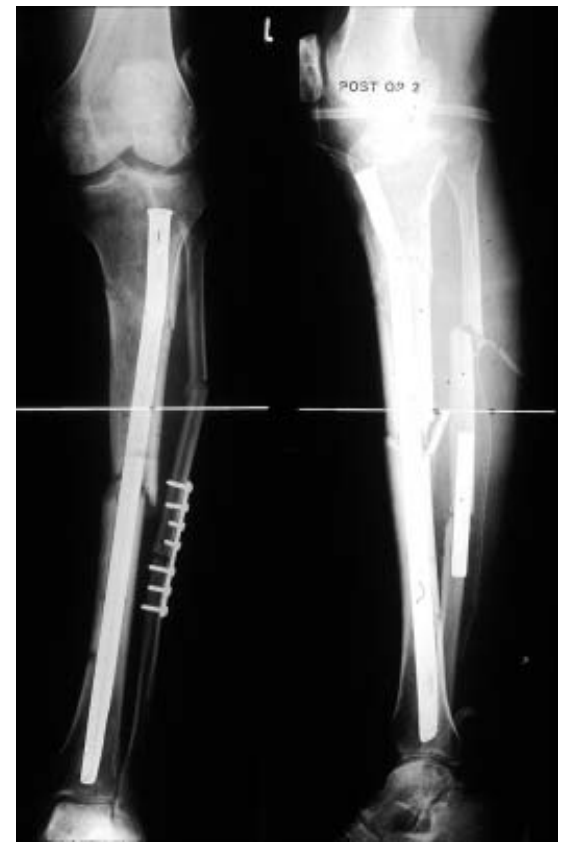

Abb.2a Varusstellung nach Marknagelung einer Unterschenkelfraktur bei zu lateralem Insertionspunkt des Nagels.

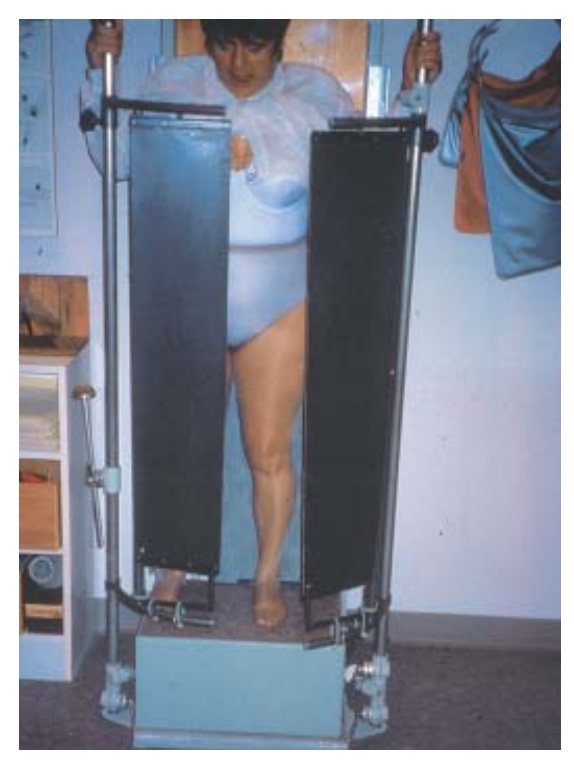

Abb.3 Anfertigung der Ganzbeinaufnahme.

Mit einer besonderen Problematik sind die in Fehlstellung verheilten Unterschenkelfrakturen mit lnfektverlauf anzusehen, da hier häufig eine Kombination von schlechter Weichteildeckung mit reduzierter Knochenqualität einhergeht.

Posttraumatische Fehlstellungen des Unterschenkels führen im Rahmen der Veränderung der Gesamtbelastungsachse

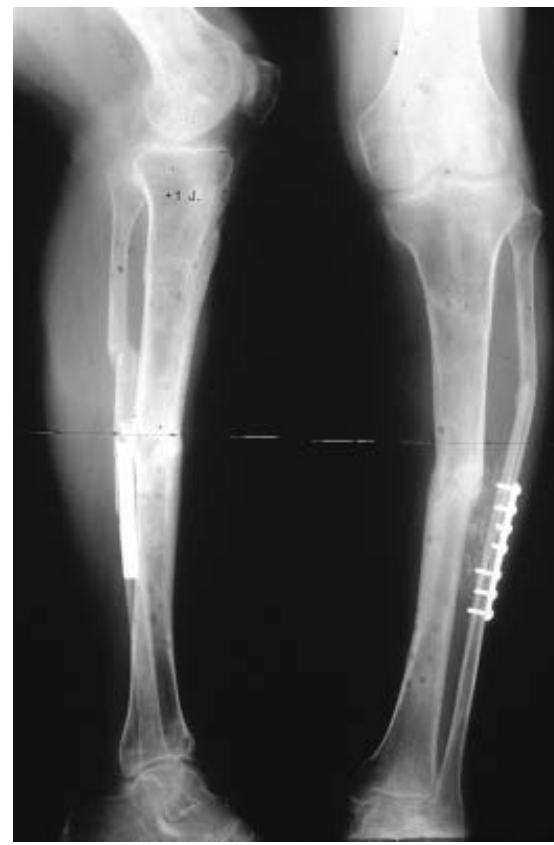

Abb.2b Marknagelentfernung nach sechs Monaten bei verheilter Fraktur.

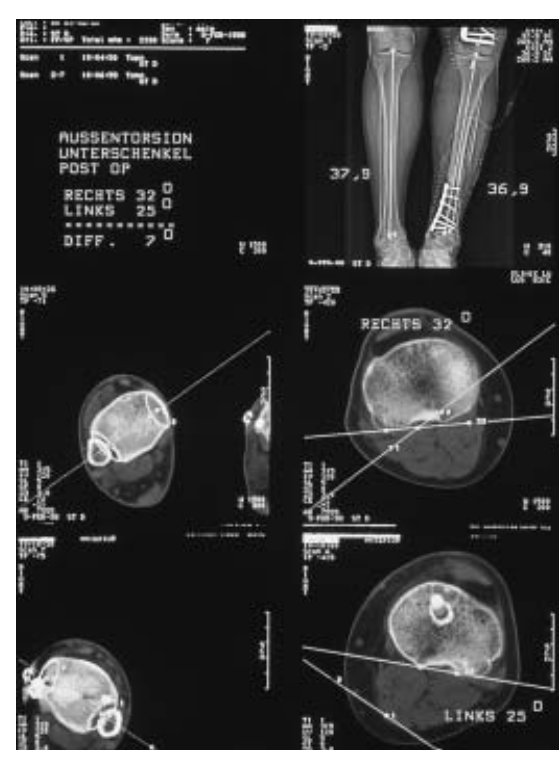

Abb. 4 Rotations-CT nach bereits durchgeführter Torsionskorrektur und verbliebener Torsionsdifferenz von $7^{\circ}$.

des Beines zur mechanischen Fehlbelastung im Knie- und Sprunggelenk mit entsprechenden Beschwerden und $\mathrm{Ar}$ throseausbildung im weiteren Verlauf. Nur die Korrektur der knöchernen Fehlstellung kann diesen natürlichen Verlauf beeinflussen, oder zum Beispiel im Bereich des Schienbeinkopfes bei Teilzerstörung der Gelenkfläche diesen Prozess zumindest um Jahre hinauszögern.

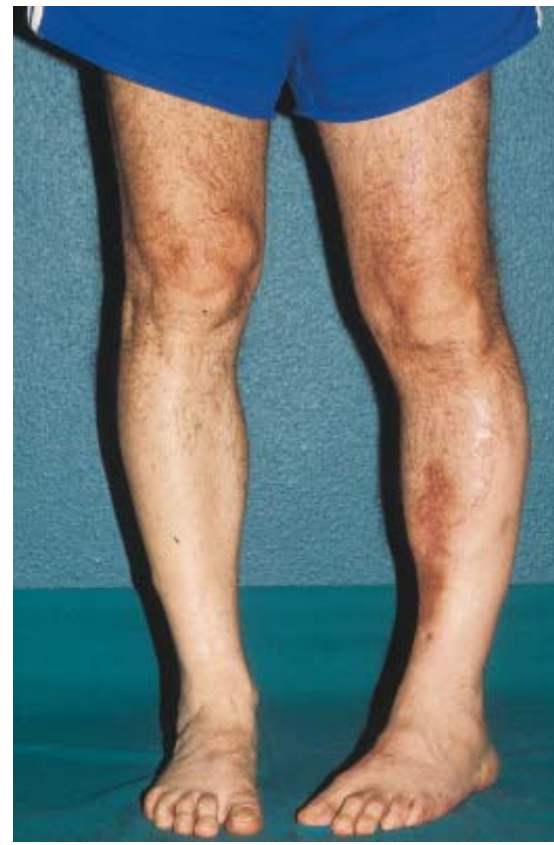

Abb.2c Klinisches Bild mit Varus- und zusätzlich vermehrter Innentorsion.

\section{Präoperative Untersuchungen}

\section{Klinische Untersuchung}

Bei Bestehen des klinischen Verdachtes oder bereits röntgenologisch nachgewiesener Fehlstellung bedarf es zur weiteren Beurteilung und Durchführung apparativer Maßnahmen der klinischen Untersuchung des gesamten Beinpaares. Die Untersuchung erfolgt sowohl im Stehen, dynamisch im Gehen, als auch im Liegen. Beide Beinachsen werden betrachtet, vorausgegangene OP-Narben sowie die gesamte Weichteilbedeckung des Unterschenkels beurteilt. Die Anamnese mit Art und Anzahl vorausgegangener Eingriffe sowie stattgehabter Komplikationen ist zu erfragen. Es muß einem bewusst sein, dass durch die klinische Untersuchung nur gröbere Abweichungen feststellbar sind, durch die klinische Untersuchung jedoch die Indikation zu weiteren bildgebenden Verfahren festgelegt wird. Achsabweichung sind möglich in der Frontalebene (Varus/-Valgus), in der Sagittalebene (Ante-/Rekurvation) sowie in der Länge (Verkürzung, Verlängerung) und Drehung (lnnen-/Außendrehung). Auch eine Fehlheilung in der longitutinalen Achsausrichtung als Translation ist möglich, sie ist jedoch als alleinige Fehlstellung selten korrekturbedürftig und wird in der Regel nur bei der Korrektur komplexerer Fehlstellungen mit korrigiert. 


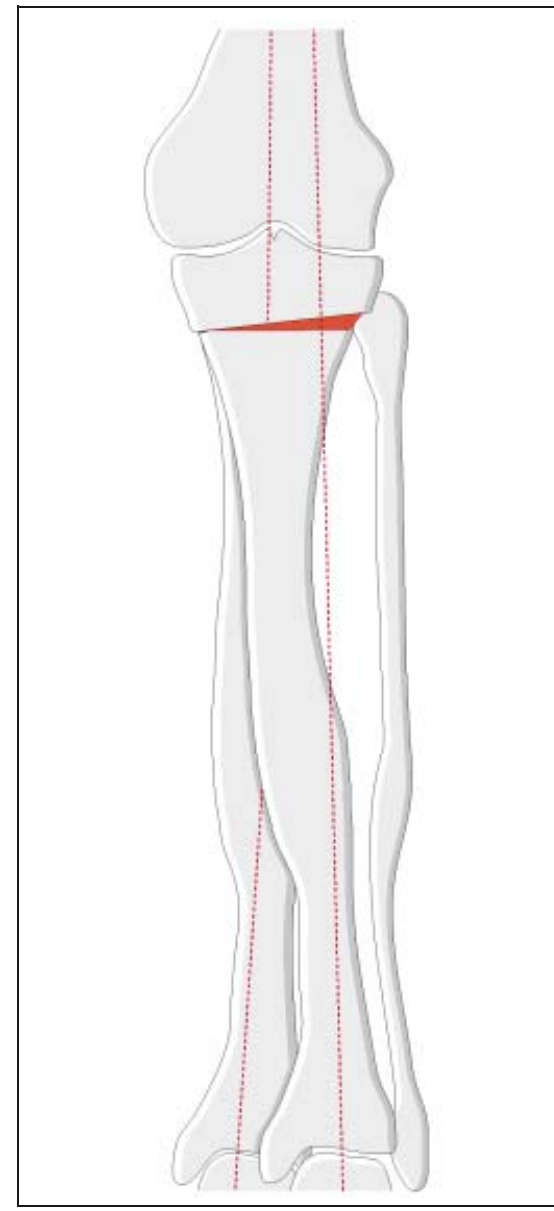

Abb.5 Zeichnerische Planung einer subtraktiven Tibiakopfosteotomie nach 20 Jahre zurückliegender fehlverheilter Unterschenkelfraktur und Ausbildung einer Varusgonarthrose.

Die Torsionsverhältnisse am Unterschenkel können sowohl in Rücken- als auch Bauchlage untersucht werden. In Rückenlage werden Hüft- und Kniegelenke rechtwinklig gebeugt, das Sprunggelenk wird in Nullstellung gehalten. Innenund Außentorsionsdifferenzen können somit gut beurteilt werden. Alternativ findet die Untersuchung in Bauchlage statt, Knie- und Sprunggelenke werden vom Untersucher rechtwinklig gebeugt, der Fuß dient als Zeiger der Rotation.

Bei Untersuchung der Unterschenkelachsen können Instabilitäten der Kniekollateralbänder Deformitäten im Valgus- oder Varussinn vortäuschen, deshalb gehören Stabilitätsuntersuchungen des Kniegelenkes unbedingt zur klinischen Untersuchung.

Streckdefizite im Hüft- und Kniegelenk können ebenso Deformitäten vortäuschen. Ein Genu antekurvatum täuscht

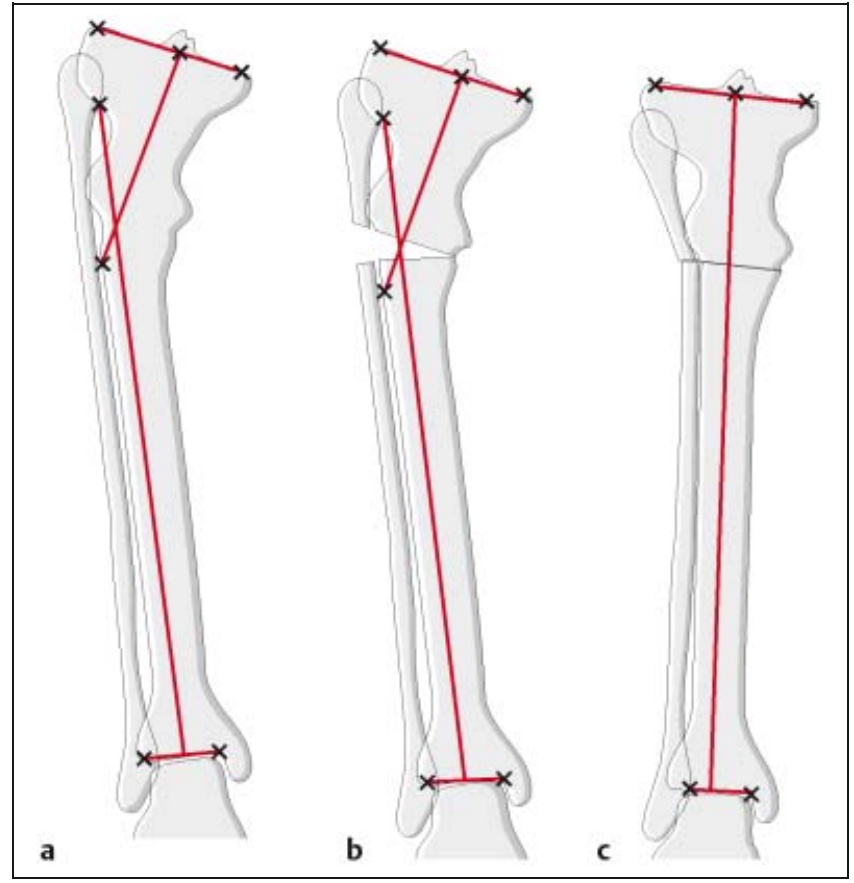

Abb. 6 Einfache Fehlstellung mit eingezeichneten Teilachsen des Unterschenkels.

Abb. 7a Additive laterale Tibiakopfosteotomie.

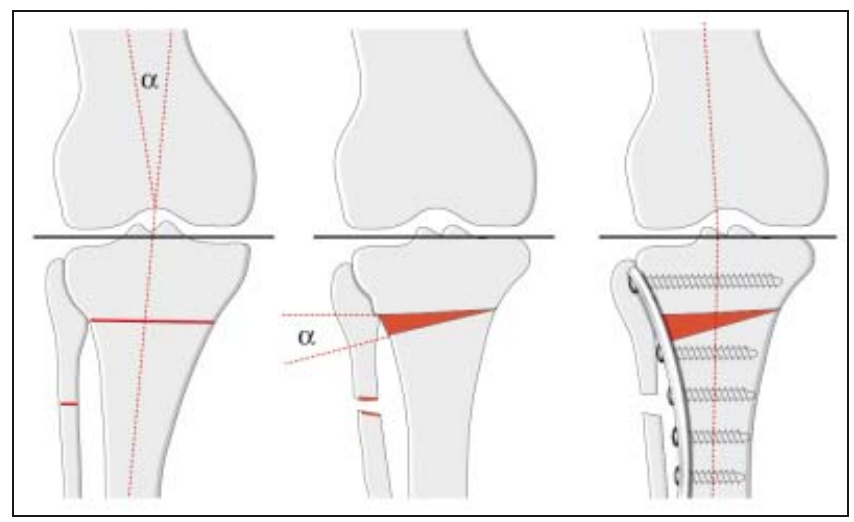

Abb. $\mathbf{7 b}$ Subtrakti-

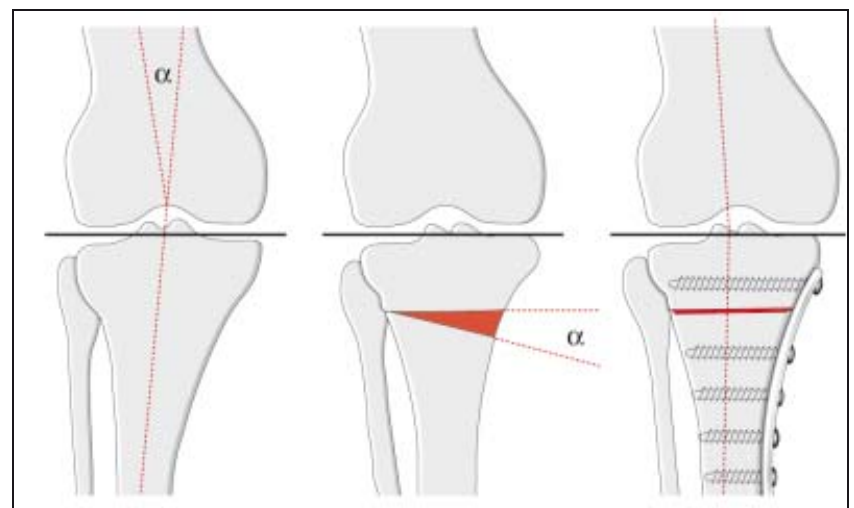

ve mediale Tibiakopfosteotomie. eine X-Beinstellung, ein Genu rekurvatum eine O-Beinstellung vor. Genauso kann eine in Außendrehstellung verheilte Unterschenkelfraktur klinisch eine vermehrte Valgusstellung vortäuschen und umgekehrt eine Innendrehung eine vermehrte Varusstellung (5) (Abb.2a-c).

\section{Bildgebende Verfahren}

\section{Nativdiagnostik}

Prinzipiell gehören zur weiteren Diagnostik die Durchführung von Röntgenganzbeinaufnahmen der verletzten und unverletzten Seite. Die Aufnahmen müs- 


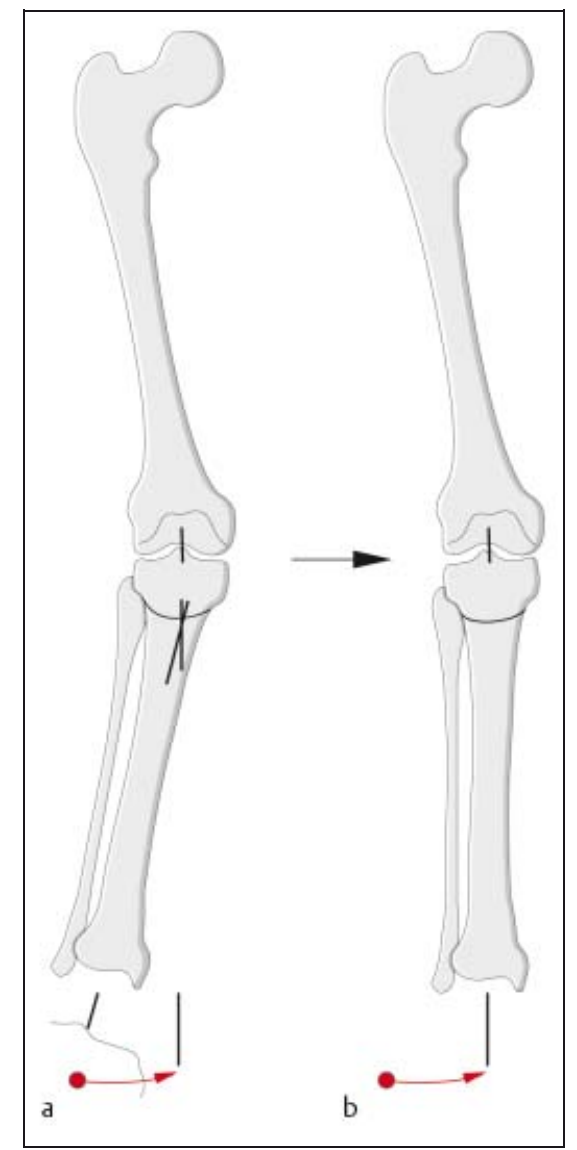

Abb. 8 Prinzip der Pendelosteotomie.

sen unter Belastung im Stehen erfolgen, die Patellaspitze muß nach vorne ausgerichtet sein. Auch die seitliche Ebene ist unverzichtbar (Abb.3).

\section{Weiterführende Diagnostik}

Da die klinische Messung der Torsionsverhältnisse allenfalls den Wert einer Schätzung hat, die für Planung und Umsetzung einer Korrekturosteotomie nicht ausreichend ist, wird eine valide und reproduzierbare Messmethode benötigt, wie sie die Computertomographie darstellt. Eine entsprechende Grafiksoftware ist erforderlich. Grundlage einer artefaktfreien Messung ist eine konstante und seitengleiche Lagerung beider Beine. Neben den Torsionsverhältnissen ergibt sich auch eine exakte Längenmessung als Nebenprodukt (Abb.4).

\section{Indikationsstellung}

Zur Indikationsstellung einer Korrekturosteotomie nach fehlverheilter Fraktur am Unterschenkel gehört sowohl die Kenntnis der physiologischen Achsenverhältnisse (siehe hierzu „Allgemeine Anmerkungen zur Indikation“ von G. Hier- holzer), als auch die Grenzen physiologischer Belastbarkeit.

Die Auswertung der angefertigten Röntgenaufnahmen, ggf. ergänzt durch weiterführende Diagnostik, zeigt Ort und Ausmaß einer evtl. Fehlstellung. Folgende Faktoren bestimmen vorrangig die Indikation zur Korrektur: [3,5]

1. die gelenkmechanische Fehlbelastung

2. der funktionelle Gesichtspunkt

3. die Auswirkung der Fehlstellung auf den Kapsel-Band-Apparat von Knieund Sprunggelenk

4. der Zustand von Knochen-, Knorpelund Weichteilen

5. die subjektiven Beschwerden

6. die kosmetischen Auswirkungen

Die Schrägstellung der Kniebasislinie von über $10^{\circ}$ korrespondierend in der Sprunggelenksebene von mehr als $10^{\circ}$ sollten vermieden werden. Verbliebene Fehlstellungen im Unterschenkelschaft sollten ab $10^{\circ}$ im Varussinne, $15^{\circ}$ im Valgussinne korrigiert werden $[1,6]$. Die Varusstellung am Unterschenkel hat eine höhere Morbidität als eine entsprechende Valgusfehlstellung, da bereits bei normalen Belastungsverhältnissen mindestens $60 \%$ der Belastung über das mediale Kniegelenkkompartiment laufen.

Sagittale Achsabweichung (Ante- oder Rekurvation) sollten ab $15^{\circ}$ korrigiert werden. Längendifferenzen können bis $3 \mathrm{~cm}$ schuhorthopädisch versorgt werden. Darüber hinaus ergibt die Kallusdistraktion eine gute Wiederherstellung der ursprünglichen Längenverhältnisse.

Supramalleolär und kniegelenksnah kann ein Valgus von $5^{\circ}$, ein Varus von allenfalls $10^{\circ}$ toleriert werden. Dank der vermehrten Supinationsmöglichkeit im unteren Sprunggelenk kann eine Varusfehlstellung besser kompensiert werden als eine Valgusfehlstellung.

Bei einer intraindividuellen Torsionsdifferenz von $15^{\circ}$ am Unterschenkel ist eine Korrektur oberhalb dieses Grenzwertes angezeigt.

Diese Richtgrößen gelten allenfalls für isolierte Fehlstellungen. Bei mehrdimensionalen Fehlstellungen müssen diese Größen ggf. enger gefaßt werden.

Um spätere Enttäuschungen zu vermeiden, müssen mit den Patienten die erreichbaren Ziele klar besprochen werden, dies auch unter dem Hinblick auf mögliche Komplikationen mit einer inadäqua- ten Korrektur von bis zu $20 \%$, einer Pseudarthrosenrate von $1-3 \%$, Nervenverletzungen mit maximal $10 \%$, bei kniegelenksnahen Korrekturen sowie einer Infektionsrate in der Literatur von ebenfalls bis zu 8\% [4]. Auf die Möglichkeit eines Kompartmentsyndroms bei kniegelenksnahen Korrekturen muss hingewiesen werden. Patientenalter und allgemeines Risiko muss in die Überlegungen mit einbezogen werden.

Je jünger der Patient, desto großzügiger sollte die Indikation zur Korrektur einer Fehlstellung getroffen werden.

\section{Präoperative Planung}

Die Planung muß zeichnerisch vom Operateur durchgeführt werden. Sie beinhaltet sowohl den operativen Zustand sowie die Zielplanung mit der vorzunehmenden Korrektur. Die zur Fixation geplanten Implantate werden eingezeichnet.

Die Planzeichnung ist als Dokument der Krankenakte beizulegen (Abb.5).

Bei der herkömmlichen Methode werden zeichnerisch die Röntgenbilder auf Papier mit Darstellung der verschiedenen Achsen, Achsenschnittpunkte und Einzeichnung der Gelenkbasis übertragen. Notwendig für die Planung sind

1. Bestimmung der Höhe der Fehlstellung (Osteotomiehöhe)

2. Bestimmung des proximalen Traglinienanteils von der Hüftkopfmitte über die Kniegelenkmitte hinaus zur Osteotomie

3. Verbindung der Sprunggelenkmitte mit dem Schnittpunkt von Osteotomie und proximalem Traglinienanteil

Bei einfacher Fehlstellung (1 Ebene) ergibt der von den Teilachsen des Unterschenkels eingeschlossene Winkel den Korrekturwinkel vor. Er entspricht in der Regel dem Fehlstellungswinkel (Abb.6).

Kombinierte Fehlstellungen liegen vor, wenn der Achsenschnittpunkt deutlich von der röntgenologisch sichtbaren Frakturhöhe abweicht. Dies ist häufig dann der Fall, wenn die Fraktur unter Seitverschiebung verheilt ist. Die Achsenschnittpunkte ergeben die Höhe der Fehlstellung. Die Korrektur einfacher diaphysärer Fehlstellungen erfolgt idealer Weise am Ort der Fehlstellung in Höhe des Schnittpunktes der Schienbeinteilachsen. Eine Diskrepanz von Fehlstellung und Achsenschnittpunkt (in Bezug auf die Lokalisa- 

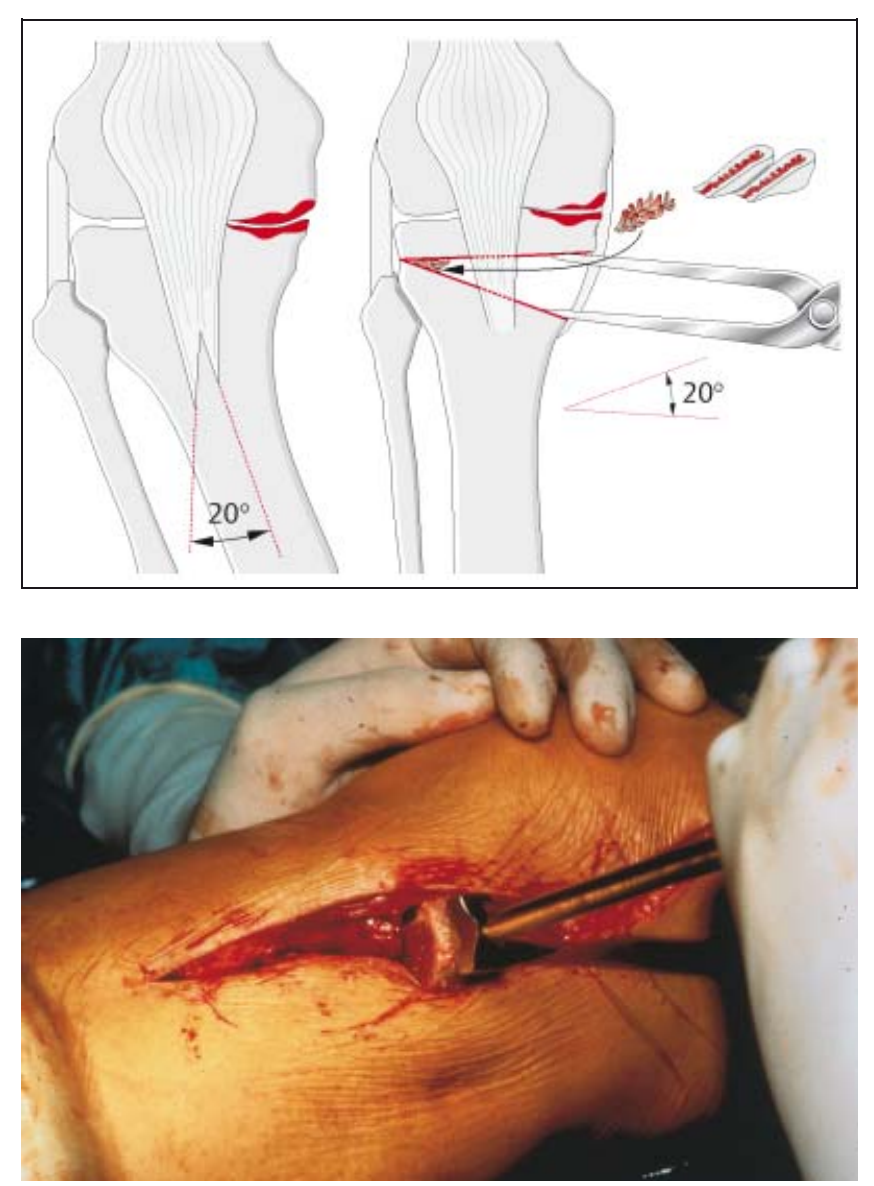

Abb. 9c Intraope-

rativer Situs.

tion) hat den am Unterschenkel häufig wichtigen Vorteil einer Osteotomie am Ort besserer Knochen- und Weichteilverhältnisse. Je weiter die Osteotomie vom Achsenschnittpunkt der Fehlstellung entfernt durchgeführt wird, um so schwieriger ist es, eine genaue Zentrierung der Traglinie bei gleichzeitiger physiologischer Lage der Gelenkbasislinien (Parallelstellung von Knie- und Sprunggelenksbasislinien) zu erreichen. Aus lokalen Gründen besteht bei in Fehlstellung verheilten Unterschenkelfrakturen häufig die Notwendigkeit, im metaphysären Bereich zu osteotomieren. Wird die Lokalisation des Achsenschnittpunktes nicht berücksichtigt und in Höhe der Fehlstellung osteotomiert, so muss neben der Angulation auch die Seitverschiebung korrigiert werden, um ein Abweichen der Traglinien zu vermeiden. Letztendlich hängt der Korrekturwinkel auch ab von der Höhe der Korrektur.

Wird die Osteotomiehöhe vom Achsenschnittpunkt nach proximal verlegt, so wird der Korrekturwinkel kleiner.

Abb. 9a Medialer Kniegelenksverschleiß bei gleichzeitig bestehender Innenbandlockerung und folgender intraligamentärer Osteotomie.

\section{Zeitpunkt von Korrektureingriffen}

Die Wahl des richtigen Zeitpunktes notwendiger Korrektureingriffe ist von vielen Faktoren abhängig.

Bei akuten Fehlstellungen, wie z.B. fehlender oder ungenügender Frakturreposition sollte abhängig von den Weichteilen zum frühstmöglichen Zeitpunkt eine Korrektur (Re-Osteosynthese) noch vor knöcherner Heilung erfolgen.

Bei den hier zu besprechenden Korrekturmaßnahmen nach knöcherner Heilung besteht zwar zur Vermeidung von Folgeschäden eine dringliche Indikation, der Eingriff kann jedoch nach optimaler Vorbereitung elektiv durchgeführt werden. Erforderliche Weichteilkonditionierungen oder Aufarbeiten von physiotherapeutischen Defiziten sollten vor der Korrektur erfolgen.

Bei bereits eingetretenen arthrotischen Veränderungen nach jahrelanger Fehlstellung ist dem Patienten eine realistische Erwartung des Erreichbaren zu vermitteln.

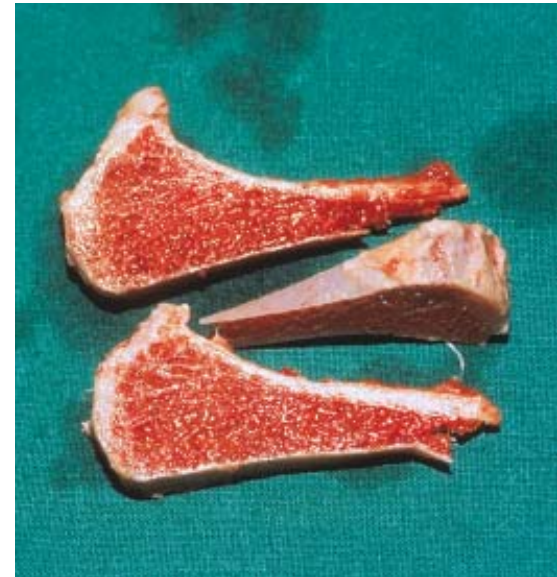

Abb.9b Beckenkammkeil.

\section{Techniken}

Prinzipiell unterscheiden wir am Unterschenkel Korrekturmöglichkeiten je nach Fehlstellung im metaphysären Bereich, knie- oder sprunggelenksnah, von diaphysär durchzuführenden Korrekturen.

\section{Kniegelenksnahe Korrekturosteotomien}

Grundsätzlich ist das Ziel jeder Korrekturosteoromie, die Wiederherstellung des Originalzustandes. Bei stattgehabten Frakturen im Bereich des Tibiakopfes mit Absinken eines Tibiaplateaus bzw. metaphysärer Fehlstellung muss jedoch in Abhängigkeit vom Arthrosegrad des Kniegelenkes zur Entlastung des geschädigten Kniegelenkskompartiments eine bewusste Überkorrektur diskutiert werden. Die meisten Korrekturosteotomien im Schienbeinkopfbereich werden zur Korrektur von Varus- oder Valgusfehlstellungen durchgeführt. Prinzipiell lassen sich subtraktive (closed wedge) Osteotomien mit Keilentfernung von additiven (open wedge) Osteotomien mit Hinzufügen entweder eines Beckenkammkeiles oder allogener Keile unterscheiden (Abb.7a u.b). Da diese Osteotomien unter Belassen der kontralateralen Kortikalis bzw. des kontralateralen Periostschlauches durchgeführt werden, sind nur eindimensionale Korrekturen möglich. Auf eine mögliche Unterschenkelverkürzung oder -verlängerung muss geachtet werden. Entsprechende Zugänge zum Tibiakopf sind von medial und lateral möglich.

Bei lateralen subtraktiven proximalen $\mathrm{Ti}$ biakopfosteotomien ist in der Regel eine Kombination mit einer Fibulaosteotomie distal der Tibiaosteotomie erforderlich 


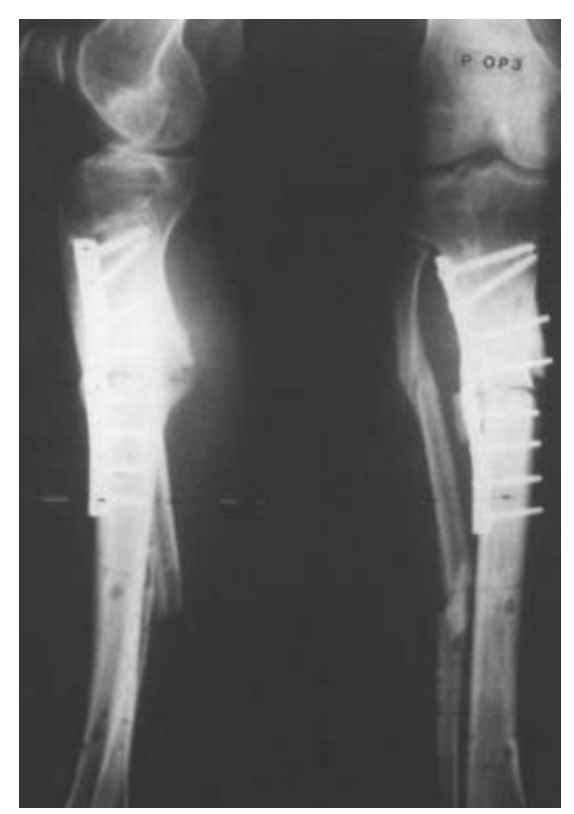

Abb.10 Korrektur Varusfehlstellung aus Abb.1b subtraktiv mittels Plattenosteosynthese.

mit der Möglichkeit einer Schädigung des Nervus peronaeus. Alternativ kann eine Lösung des tibio-fibularen Gelenkes, welches ein Höhertreten des Fibulaköpfchens erlaubt, durchgeführt werden.

An weiteren Möglichkeiten kommt eine sogenannte Dom- oder Pendelosteotomie mit bogenförmiger (domförmiger) Osteotomie in Frage (Abb. 8). Die Pendelosteo-

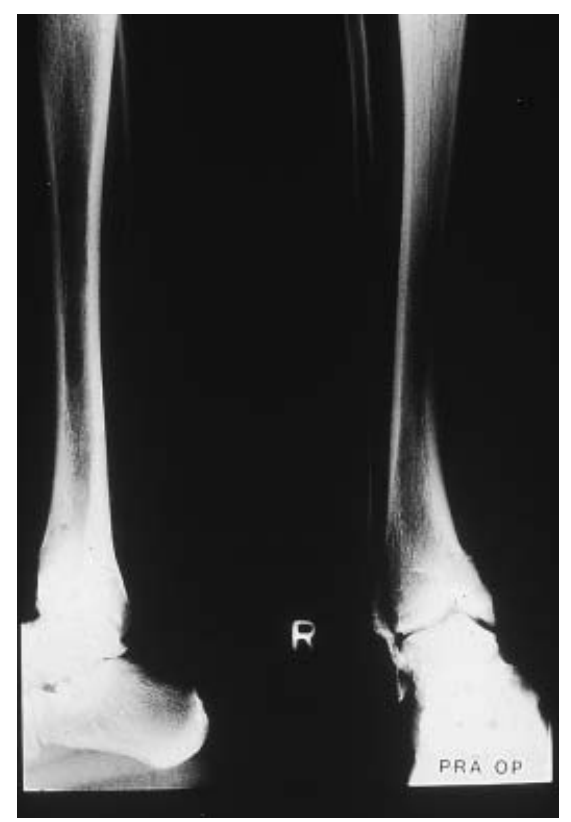

Abb.11a In Varusstellung verheilte körperferne Unterschenkelfraktur. Korrektur kontinuierlich mittels Ilisarov-Fixateur. tomie erlaubt eine genaue Korrektur und bietet eine große metaphysäre Kontaktfläche zur raschen Knochenheilung. Bei kniegelenksnahen Osteotomien ist auch auf die Spannungsverhältnisse des Bandapparates zu achten. So kann z.B. bei einer medialen Seitenbandinstabilität durch eine additive intraligamentäre Anhebeosteotomie neben der knöchernen Korrektur gleichzeitig eine Bandstraffung erfolgen. Eine Fixation dieser Osteotomie mit Einbringen eines Keils ist nicht dringend erforderlich (Abb.9a-c).

Bei all diesen Verfahren handelt es sich um einzeitige Eingriffe, also in einer Sitzung durchzuführende Verfahren. Eine Fixation ist mit allen auch zur Frakturversorgung eingesetzten Implantaten möglich, zum Beispiel mittels L-Platten oder speziellen OsteotomiePlatten (z.B. HTO-Instrumentarium). Achskorrekturen können auch kontinuierlich mittels äußerer Spanner in unilateraler Technik oder mit Ring-Fixateuren durchgeführt werden.

\section{Diaphysäre Korrekturen}

Prinzipiell birgt die Korrektur im Bereich der Diaphyse die Gefahr einer verzögerten Heilung. Additive Verfahren sind wesentlich ungünstiger. In der Regel wird bei der posttraumatisch diaphysären Fehlstellung eine subtraktive Keilosteotomie durchgeführt, auch Schrägosteotomien helfen das Risiko einer verzögerten
Knochenheilung zu mindern. Während Achsen- und Torsionsfehlstellungen prinzipiell durch alle internen Techniken, wie sie auch bei der primären Versorgung von Frakturen verwendet werden, korrigiert werden können, ist dies bei Längendiskrepanzen nur im begrenzten Maße möglich. Vorwiegend kommen an der Diaphyse Plattenosteosynthesen (Abb.10) zum Einsatz, gelegentlich auch die Marknagelung. Bei kritischen Weichteilen ist der Einsatz vom Fixateursystem als unilaterale Fixation mit Angulationsmöglichkeit oder Ringfixateursystemen gut geeignet. Die schrittweise Korrektur bietet sich an, zur Korrektur komplexer Fehlstellungen.

Bei der Korrektur komplexer Fehlstellung unter Einsatz von Ringfixateursystemen ist eine exakte Planung bez. der Drehpunkte des Fixateursystems erforderlich. Die Montage des Drehpunktes ist auch davon abhängig, ob gleichzeitig eine Längenkorrektur durchgeführt werden soll. Die Transportstrecke am Gewindestab entspricht nicht der tatsächlichen am Knochen und kann entweder rechnerisch oder zeichnerisch festgestellt werden (Abb.11a-c).

Es gilt:

Je geringer der Drehpunktabstand der Osteotomie vom Achsenschnittpunkt ist, um so genauer ist die postoperative Traglinienzentrierung im Kniegelenk.

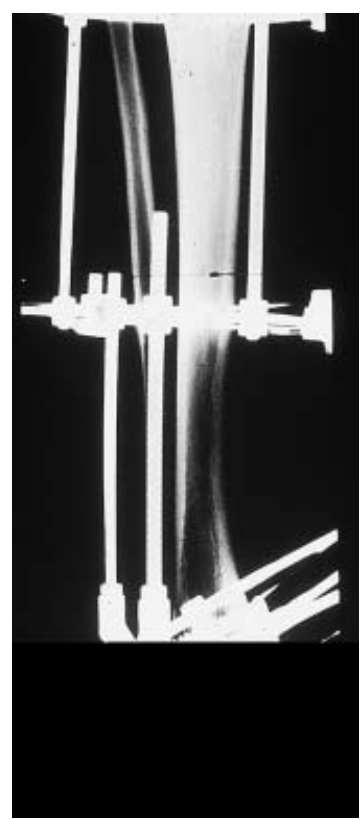

Abb.11 b Die Ringe bilden den Fehlstellungswinkel ab.

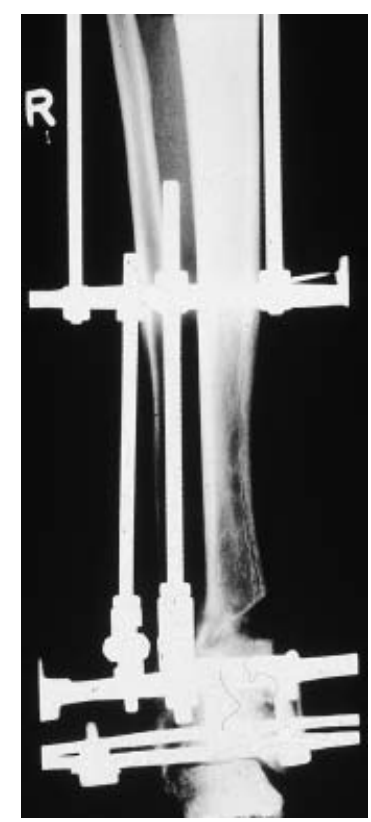

Abb.11c Nach Abschluß der Korrektur parallele Ausrichtung. 


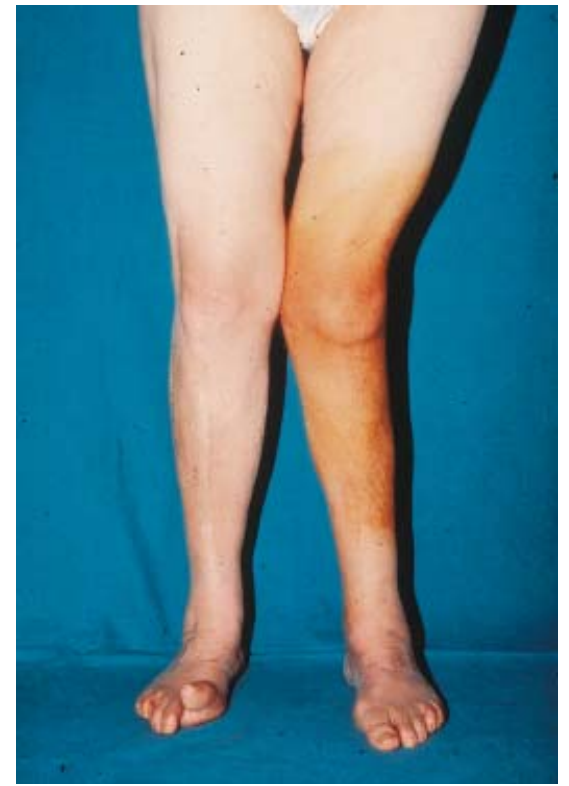

Abb.12a Valgusfehlstellung nach verheilter Tibiakopffraktur.

\section{Schlussfolgerung}

Mit einer frühen Korrektur knöcherner Fehlstellungen kann die Belastungsachse wiederhergestellt und ein Folgeschaden vermieden werden. Bei fraktur- oder fehlstellungsbedingten Gelenkschäden wird eine Entlastung des betroffenen Gelenkabschnittes erreicht, die ggf. weitere

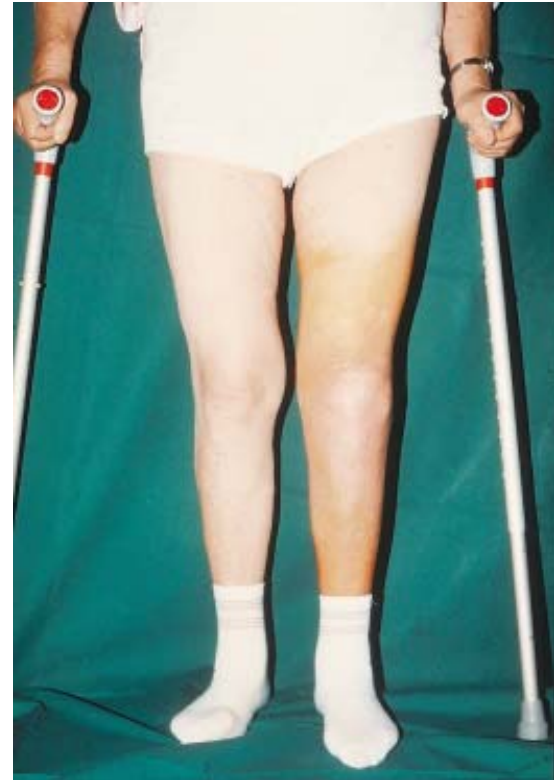

Abb.12b Ergebnis nach Korrektur.

erforderliche Gelenkeingriffe um Jahre oder sogar Jahrzehnte hinausschiebt. Voraussetzung für ein Gelingen der Korrekturoperation ist eine genaue Analyse der Fehlstellung, eine exakte präoperative Planung mit Ist/Sollvergleich und eine präzise operative Durchführung (Abb.12au.b).

\section{Literatur}

1 Braun W, Markmiller M. Müssen Achsenfehler korrigiert werden: Die Grenzen physiologischer Belastung als Indikation zur Korrekturosteotomie. Hefte zu Der Unfallchirurg 1995; 257: 653 - 663

2 Hierholzer G, Müller KFH. Korrekturosteotomien nach Trauma an der unteren Extremität. Springer 1984

3 Hierholzer G, Hax PM. Indikation zur Korrekturosteotomie bei Fehlstellungen nach Frakturen (1984) in Hierholzer G, Miller KM, Korrekturosteotomien nach Traumen an der unteren Extremität. Springer Berlin, Heidelberg 1984

4 Josten Ch. Umstellungen „rund ums Knie“ Hefte zu Der Unfallchirurg 1995; 257 : 675-680

5 Wentzensen A. Tibia und Sprunggelenk Hefte zu Der Unfallchirurg 1995; 257 : 680-686

6 Strecker W, Becker U, Hehl G, Kepller P, Kinzl L. Einzeitige Korrekturosteotomien nach kniegelenksnahen Frakturen - Grundlagen, Indikation und Operationstechniken. Posttraumatische Beindeformitäten 1997. Springer, $199-214$

\section{Dr. med. F. Holz}

Leitender Oberarzt

BG-Unfallklinik Ludwigshafen Unfallchirurgische Klinik Mannheim

\section{Dr. med. V. Heppert}

Oberarzt

Prof. Dr. med. A. Wentzensen Ärztlicher Direktor

BG-Unfallklinik Ludwigshafen Ludwig-Guttmann-Str. 13 67071 Ludwigshafen 